\title{
ARTICLE Inhibition of M2-like macrophages by all-trans retinoic acid prevents cancer initiation and stemness in osteosarcoma cells
}

Xue-jing Shao ${ }^{1}$, Sen-feng Xiang ${ }^{1}$, Ying-qian Chen ${ }^{1}$, Ning Zhang ${ }^{2}$, Ji Cao ${ }^{1}$, Hong Zhu ${ }^{1}$, Bo Yang ${ }^{1}$, Qian Zhou ${ }^{1,3}$, Mei-dan Ying ${ }^{1}$ and Qiao-jun $\mathrm{He}^{1}$

Emerging evidence indicates that M2-polarized tumor-associated macrophages (TAMs) directly participate in tumor initiation, progression and metastasis. However, to date, few studies have investigated novel strategies for inhibiting TAMs in order to overcome osteosarcoma. In this study, we reported that M2 macrophages were enriched in osteosarcoma tissues from patients, and M2-polarized TAMs enhanced cancer initiation and stemness of osteosarcoma cells, thereby establishing M2-polarized TAMs as a therapeutic target for blocking osteosarcoma formation. We also found that all-trans retinoic acid (ATRA) weakened TAM-induced osteosarcoma tumor formation by inhibiting M2 polarization of TAMs in vivo, and inhibited the colony formation, as well as sphereformation capacity of osteosarcoma cells promoted by M2-type macrophages in vitro. Furthermore, M2-type macrophages enhanced cancer stem cells (CSCs) properties as assessed by increasing the numbers of $C D 117^{+}$Stro- $1^{+}$cells accompanied by the upregulation of CSC markers (CD133, CXCR4, Nanog, and Oct4), which could clearly be reduced by ATRA. Taken together, the results of this study demonstrated the role of M2-polarized TAMs in osteosarcoma initiation and stemness by activating CSCs, and indicated that ATRA treatment is a promising approach for treating osteosarcoma by preventing M2 polarization of TAMs.

Keywords: osteosarcoma; ATRA; tumor-associated macrophages; M2 polarization; cancer initiation; stemness

Acta Pharmacologica Sinica (2019) 40:1343-1350; https://doi.org/10.1038/s41401-019-0262-4

\section{INTRODUCTION}

Osteosarcoma (OS), the most common type of bone cancer, is the second leading cause of cancer-related death in children and adolescents [1]. Despite intensive chemotherapy, the survival rate of patients with osteosarcoma has not changed over the past 30 years, mainly due to metastasis and recurrence [2]. Thus, novel strategies to further prolong the survival of osteosarcoma patients are urgently needed.

Cancer stem cells (CSCs), also known as tumor-initiating cells or tumor-propagating cells, constitute a biologically unique subset of stem-like cells within the bulk tumor cell population [3, 4]. Emerging evidence indicates the presence of CSCs in osteosarcoma, and the inadequacy of current treatments may result from an inability to effectively target these $\operatorname{CSCs}[5,6]$. The clinical implications of the CSC model suggest that only the elimination of CSCs will result in eradication of the tumor because uneliminated CSCs will inevitably lead to tumor relapse. CSCs are highly resistant to chemotherapy and radiotherapy [7]. Consequently, the concept of eliminating CSCs as a therapeutic strategy is already beginning to revolutionize the approach to cancer treatment. However, there is still a lack of research into novel strategies for eliminating CSCs to eradicate osteosarcoma. The importance of the tumor microenvironment in promoting cancer initiation and tumor growth has also been increasingly recognized over the past decade $[8,9]$. Many studies have provided evidence that the tumor microenvironment is essential for the functions of CSCs [10-12].

Tumor-associated macrophages (TAMs) serve as a source of key components in tumor microenvironments and have been reported to trigger tumorigenicity and tumor progression by directly communicating with CSCs to promote their stemness and/ or subsequent oncogenic properties $[13,14]$. TAMs exist as two polarized phenotypes, classically activated macrophages (M1) and alternatively activated macrophages (M2). TAMs predominantly display an M2 phenotype in tumors, and M2-type TAMs have been reported to promote cancer stem cell-like properties in several cancers, including hepatocellular carcinoma [15], breast cancer [16], non-small cell lung cancer [17], pancreatic ductal adenocarcinoma [18], and glioblastoma multiforme [19]. Therefore, targeting M2-TAMs to interfere with CSCs might provide a novel approach for osteosarcoma therapy. All-trans retinoic acid (ATRA), the active derivative of vitamin A, was shown to effectively skew macrophages away from $\mathrm{M} 2$ polarization in our previous study [20]. Thus, we sought to determine whether M2-type TAMs could regulate osteosarcoma stem cells and whether ATRA could prevent cancer initiation and stemness by inhibiting M2 polarization of TAMs in osteosarcoma.

In this study, we found that osteosarcomas were infiltrated with $\mathrm{CD}_{209^{+}}$M2-type TAMs and that these M2-polarized TAMs promoted osteosarcoma progression by activating CSCs. We

\footnotetext{
${ }^{1}$ Institute of Pharmacology and Toxicology, Zhejiang Province Key Laboratory of Anti-Cancer Drug Research, College of Pharmaceutical Sciences, Zhejiang University, Hangzhou 310058, China; ${ }^{2}$ Department of Orthopedics, The Second Affiliated Hospital of Zhejiang University, Zhejiang University, Hangzhou 310009, China and ${ }^{3}$ Department of Pharmacy, Hangzhou Medical College, Hangzhou 310053, China

Correspondence: Qian Zhou (qianz1220@hmc.edu.cn) or Mei-dan Ying (mying@zju.edu.cn) or Qiao-jun He (qiaojunhe@zju.edu.cn)
}

Received: 24 January 2019 Accepted: 22 May 2019

Published online: 11 July 2019 
also found that ATRA effectively weakened TAM-induced osteosarcoma tumor formation in vivo by decreasing CSC activity and thus inhibited the colony-forming as well as the sphere-forming capacity of osteosarcoma cells promoted by M2type macrophages in vitro. In conclusion, our results demonstrated that M2-polarized TAMs were associated with osteosarcoma initiation and that ATRA may be a promising agent to interfere with CSCs by blocking M2 polarization of TAMs during osteosarcoma treatment.

\section{MATERIALS AND METHODS}

Materials

Recombinant mouse IL-13, mouse IL-4, mouse EGF, mouse FGF, and M-CSF were purchased from Peprotech (USA), and N2 was supplied by Life Technologies (USA). ATRA was purchased from Sigma-Aldrich (MO, USA). DMEM and fetal bovine serum (FBS) were purchased from Gibco (USA). For flow cytometry analysis, antibodies, including monoclonal anti-Stro-1, anti-PE-CD117, and anti-PE-CD209 antibodies, were purchased from Sigma-Aldrich (USA), BD Biosciences (USA), and eBioscience (USA), respectively. For immunofluorescence, primary antibodies, including antibodies against mouse CD209 and human CD209 and F4/80, were purchased from eBioscience (USA) and Biolegend (USA), respectively, while secondary antibodies were purchased from Life Technologies (USA).

Cell lines and cell culture

RAW264.7 and K7M2 WT cells were obtained from the Cell Bank of the Chinese Academy of Sciences (Shanghai, China) and maintained in DMEM containing $10 \%$ FBS. Both cell lines were maintained at $37^{\circ} \mathrm{C}$ in a humidified atmosphere containing $5 \% \mathrm{CO}_{2}$.

Immunohistochemistry (DAB staining)

Paraffin-embedded tissue sections were dewaxed, rehydrated, and microwaved in pH 9.0 Tris-EDTA buffer for CD209 staining. Then, a Histostain-Plus Kit was used following the manufacturer's instructions. The immunohistochemical staining patterns of CD209 were evaluated by an experienced pathologist and scored as follows: (1) negative ("-", no positive staining or up to $1 \%$ scattered positive cells); (2) low (" + ", heterogeneous staining, with an area corresponding to at least $20 \%$ of the section showing $2 \% \sim 10 \%$ positive cells); (3) medium (" ++ ", heterogeneous, with at least $20 \%$ of the section showing $10 \% \sim 50 \%$ positive cells); and (4) high $("+++$ ", variable to almost homogeneous staining, with at least $20 \%$ of the section showing $51 \% \sim 90 \%$ positive cells).

\section{Orthotopic transplantation}

Four- to five-week-old female BALB/c mice (National Rodent Laboratory Animal Resource, Shanghai, China) were used for all experiments. The Animal Research Committee at Zhejiang University approved all animal studies, and animal care was provided in accordance with institutional guidelines. For tumor formation, K7M2 WT cells $\left(1 \times 10^{5}\right)$ mixed with or without conditioned macrophages (1:3) were administered via intraosseous injection into the proximal tibia and distal femur [21]. The mice were divided into four groups: the K7M2 WT group; the K7M2 WT + ATRA-treatment group; the K7M2 WT + RAW group; and the K7M2 WT + RAW + ATRA-treatment group. The mice were treated i.g. daily for 7 days with $20 \mathrm{mg} / \mathrm{kg}$ ATRA before K7M2 WT cell injection; after inoculation, the mice were treated i.g. daily for 7 days for 4 consecutive weeks with $40 \mathrm{mg} / \mathrm{kg}$ ATRA. All animal work was conducted with the approval of the Animal Care and Use Committee of the National Cancer Institute.

\section{Immunofluorescence}

Immunofluorescence analysis of the tumor tissues was performed as previously described [22]. Briefly, cryostat sections were fixed and permeabilized. Primary antibodies against F4/80 and CD209 were used, followed by staining with secondary antibodies conjugated to Alexa Fluor 488 or 594 . Nuclei were visualized by DAPI staining. Then, the $\mathrm{F} 4 / 80^{+}$area (\% of tumor area) and the CD209 ${ }^{+}$area (\% of tumor area) were analyzed using ImageJ software.

Bone marrow-derived macrophage (BMDM) isolation and differentiation

Bone marrow-derived macrophage (BMDM) isolation was performed as previously described [23]. Briefly, C57BL/6 mice were euthanized, and the femurs were dissected using scissors to obtain bone marrow cells. Then, the bone marrow cells were cultured in DMEM containing $10 \% \mathrm{FBS}$ and incubated with M-CSF (Cell Signaling Technology, $50 \mathrm{ng} / \mathrm{mL}$ ) for 3 days to obtain BMDMs.

Conditioned medium (CM) preparation

Macrophage polarization was induced by culturing cells with IL-13 $(20 \mathrm{ng} / \mathrm{mL})$ or IL-4 $(20 \mathrm{ng} / \mathrm{mL})$ for $48 \mathrm{~h}$. Where indicated, ATRA was added during macrophage polarization. Then, the IL-13/IL-4/ATRAcontaining medium was replaced with serum-free normal medium. The differently polarized macrophages were incubated in serum-free normal medium for $24 \mathrm{~h}$, after which culture supernatants were collected as CM. Notably, there was no IL-13/IL-4/ATRA in the CM. The CM was centrifuged at $2000 \mathrm{r} / \mathrm{min}$ to precipitate debris and stored at $-80^{\circ} \mathrm{C}$. For stimulation with RAW264.7-CM (RM) or BMDMCM (BM), K7M2 WT cells were supplemented with RAW264.7-CM or BMDM-CM at a final concentration of $50 \%$ (vol/vol).

Flow cytometry

Samples were incubated with anti-PE-CD209, anti-CD117 and antiStro-1 antibodies according to the manufacturer's instructions. For each sample, at least $1 \times 10^{4}$ cells were analyzed using a BD FACSCalibur flow cytometer.

\section{Reverse transcription PCR}

Quantitative real-time RT-PCR analysis was performed by using TAKARA SYBR Premix EXTaq ${ }^{\text {TM }}$ as previously described [24]. Reaction mixtures containing SYBR Green were prepared following the manufacturer's protocol. The sequences of the primers used for quantitative RT-PCR were as follows: TGF-beta, forward: 5'-GGTGGTATACTGAGACACCTTG-3' and reverse: 5'-CCCAAGGAAA GGTAGGTGATAG-3'; FIZZ1, forward: 5'-GCTGATGGTCCCAGTGAA TA-3' and reverse: 5'-CGTTACAGTGGAGGGATAGTTAG-3'; CD133, forward: 5'-GCTITGCAATC TCCCTGTTG-3' and reverse: 5'-TTGATC CGGGTTCTTACCTG-3'; CXCR4, forward: 5'-GGTGGTCTATGTTGGCG TCT-3' and reverse: 5'-TGGAGTGTGACAGCTTGGAG-3'; Nanog, forward: 5'-AAAGAATCTTCACCTATGCC-3' and reverse: 5'-GAAGGA AGAGGAGAGACAGT-3'; Oct4, forward: 5'-CTGGAGAAGGAGAAGCT GGA-3' and reverse: 5'-CAAATTGCTCGAGTTCTTCTG-3'; and ACTIN, forward: 5'-GGTCATCACTATTGGCAACG-3' and reverse: 5'-A CGGAT GTCAACGTCACACT-3'.

\section{Colony formation assays}

A total of 2500 cells per well were seeded in six-well plates and incubated with CM for 7 days. The colonies were then fixed with $2 \%$ formaldehyde and stained with $0.5 \%$ crystal violet. Images were acquired, and the colonies in each well were counted.

Sphere-formation assay

A total of 1000 living osteosarcoma cells per well were seeded in an ultra-low attachment 6-well microplate (Corning Incorporated, Corning, NY, USA) as previously described [25] and cultured in serum-free CM supplemented with $20 \mathrm{ng} / \mathrm{mL}$ EGF, $20 \mathrm{ng} / \mathrm{mL}$ FGF and N2 (1:100; Life Technologies) for 2 weeks. Cell spheroids were visualized in a low-power microscopic field.

Statistical analysis

For all parameters measured, the values for all samples under the different experimental conditions were averaged, and the SD was 
calculated. The statistical significance of differences between groups was determined with Student's unpaired two-tailed $t$-test. One-way ANOVA followed by Tukey's multiple comparisons test was used to determine the differences among multiple pairs.

\section{RESULTS}

M2 macrophages are enriched in osteosarcoma tissues from patients First, to study the enrichment of M2-type macrophages in osteosarcoma, we referred to two data sets, GSE12865 and GSE14359. The data showed that the expression levels of three classical M2 macrophage markers-CD163, MRC1, and CCR2were all markedly higher in human osteosarcoma (HOS) tissue samples than in normal human osteoblasts (HOBs) (Fig. 1a, b). Next, we evaluated the expression level of CD209 (another M2 macrophage marker) in primary osteosarcoma tissues from patients by an immunohistochemistry assay. A total of 14 separate osteosarcoma tissue blocks were analyzed, and the intensities and percentages of staining were determined. The staining results for representative cases with different CD209 expression levels are shown in Fig. 1c. Furthermore, the distribution patterns of CD209 in all patient samples were analyzed: only 3 of the 14 osteosarcoma samples (21.43\%) exhibited negative expression of CD209 in tumor tissues, whereas the remaining 11 samples (78.57\%) showed mild to intense CD209 immunoreactivity (Fig. 1d). Consequently, these results demonstrated that $\mathrm{M} 2$ macrophages were enriched in human osteosarcoma tissues.

M2 macrophages contribute to osteosarcoma initiation and progression

Since M2 macrophages were enriched in osteosarcoma tissues, we were prompted to study the role of macrophages in osteosarcoma initiation. We first injected K7M2 WT osteosarcoma cells with RAW264.7 macrophage cells into the tibia of mice and then examined tumor initiation. Tumors in mice transplanted with K7M2 WT and RAW264.7 cells showed faster growth properties

\section{a GSE12865}
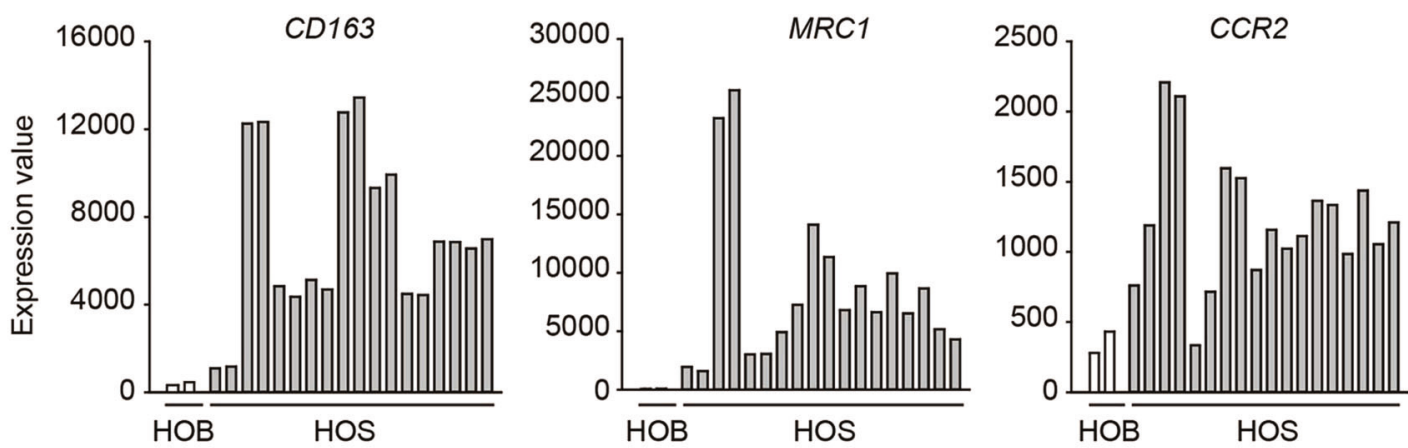

\section{b GSE14359}
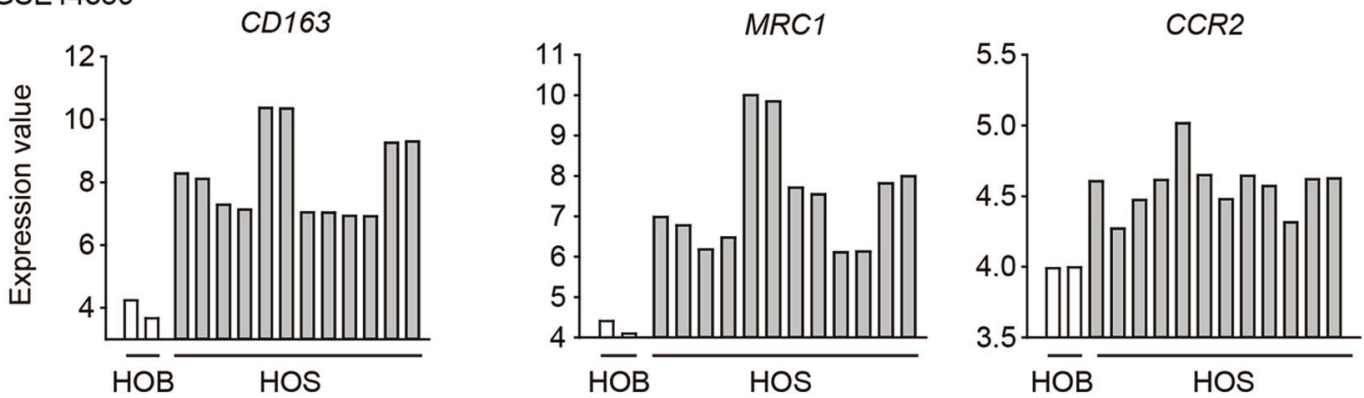

C
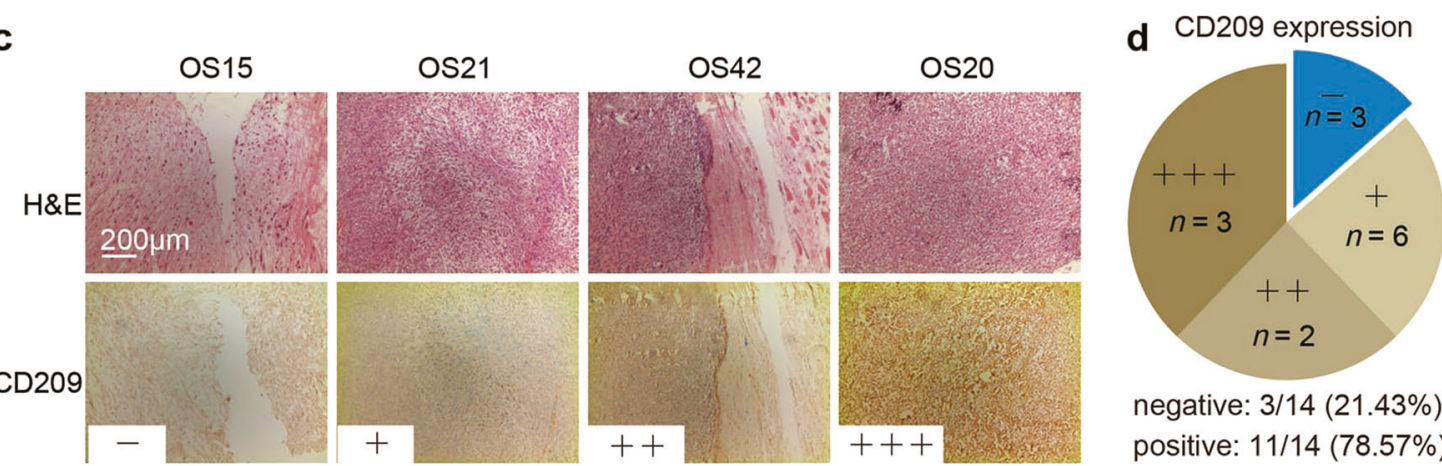

negative: $3 / 14(21.43 \%)$

positive: $11 / 14(78.57 \%)$

Fig. 1 Enrichment of M2 macrophages in osteosarcoma tissues. a, b The expression of CD163, MRC1 and CCR2 in normal human osteoblasts and human osteosarcoma tumors from the GSE12865 (a) and GSE14359 (b) data sets. HOB: normal human osteoblasts; HOS: human osteosarcoma tumor. c Immunohistochemical analysis of four representative human osteosarcoma tissues to assess the CD209 expression levels. The four samples were subjected to immunohistochemical staining using an anti-CD209 antibody, and the cryosections were then stained with H\&E. d The expression levels of CD209 in 14 osteosarcoma tumor samples were graded and summarized in pie charts. c, d "_": negative expression; "+": low expression; "++": moderate expression; and "+++": high expression 
1346

than those in control mice transplanted with only K7M2 WT cells (Fig. 2a). Then, we weighed the legs harboring the tumors; as shown in Fig. 2b, the legs harboring tumors in mice transplanted with both K7M2 WT and RAW264.7 cells were heavier than those of control group mice. Furthermore, histological examination showed that compared with mice transplanted with only K7M2 WT cells, mice transplanted with both K7M2 WT and RAW264.7 cells were enriched in M2-like TAMs $\left(\mathrm{F} 4 / 80^{+} \mathrm{CD} 209^{+}\right)$(Fig. 2c).
These results demonstrated that $\mathrm{M} 2$ macrophages could promote osteosarcoma initiation and progression.

ATRA delays the initiation of osteosarcoma induced by M2-polarized TAMs

We then sought to determine whether ATRA could inhibit osteosarcoma initiation by impacting the polarization of macrophages. The results showed that the ATRA-treated group had a

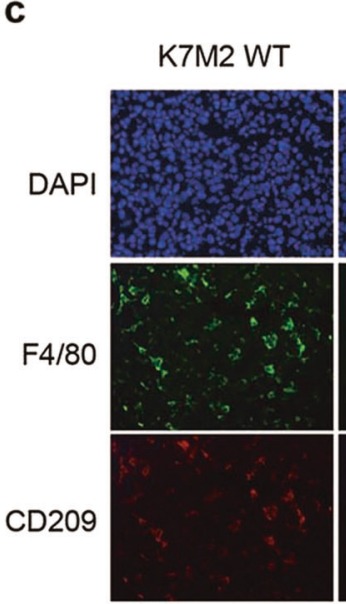

C

K7M2 WT+RAW+ATRA
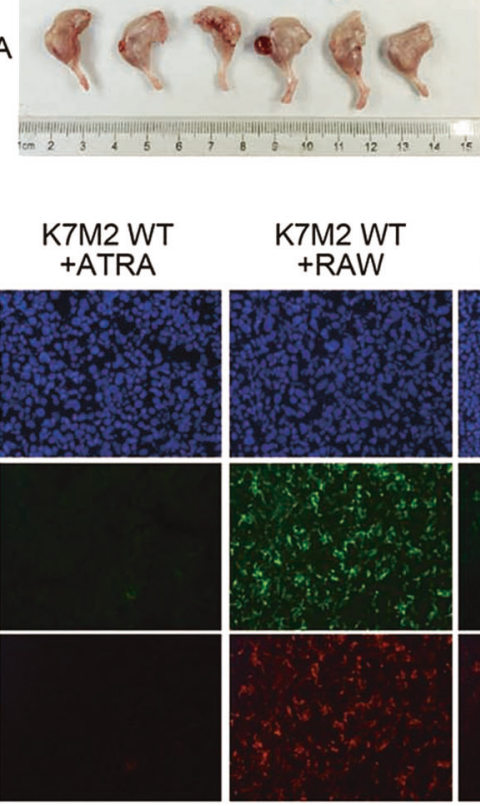

b

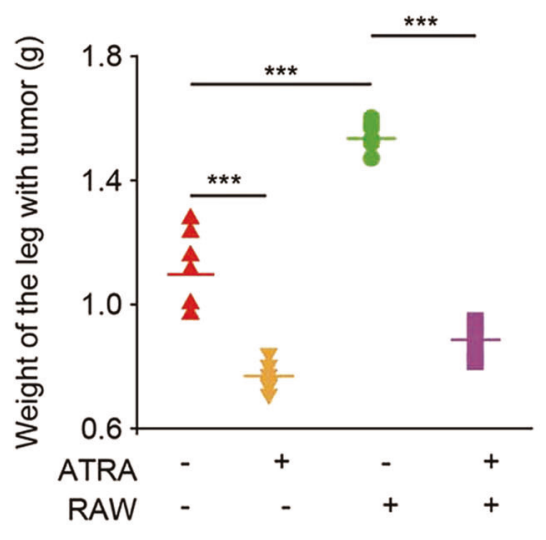

7M2 WT

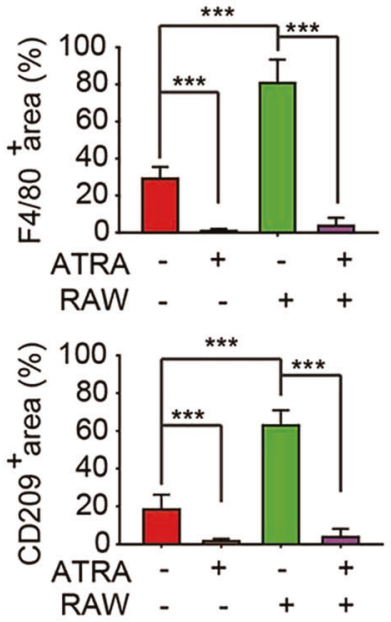

\begin{tabular}{|c|c|c|c|c|}
\hline & K7M2 WT & K7M2 WT+ATRA & K7M2 WT+RAW & K7M2 WT+RAW+ATRA \\
\hline WBC $\left(10^{9} / L\right)$ & $3.31 \pm 1.02$ & $3.89 \pm 1.49$ & $3.93 \pm 1.30$ & $4.08 \pm 2.52$ \\
\hline$\%$ NEUT & $25.44 \pm 7.05$ & $31.60 \pm 4.22$ & $20.65 \pm 4.66$ & $37.15 \pm 13.55$ \\
\hline$\%$ LYMPH & $56.22 \pm 14.20$ & $47.48 \pm 5.89$ & $62.82 \pm 10.68$ & $45.70 \pm 19.21$ \\
\hline \%MONO & $14.50 \pm 9.33$ & $17.54 \pm 4.22$ & $13.90 \pm 5.94$ & $14.73 \pm 5.46$ \\
\hline$\%$ EOS & $2.98 \pm 3.51$ & $2.54 \pm 1.02$ & $2.10 \pm 0.84$ & $1.40 \pm 1.39$ \\
\hline$\%$ BASO & $0.60 \pm 0.63$ & $0.66 \pm 0.63$ & $0.35 \pm 0.12$ & $0.50 \pm 0.27$ \\
\hline $\operatorname{RBC}\left(10^{12} / L\right)$ & $6.47 \pm 2.65$ & $8.04 \pm 0.99$ & $9.15 \pm 0.44$ & $7.39 \pm 0.80$ \\
\hline $\mathrm{HGB}(\mathrm{g} / \mathrm{dL})$ & $8.82 \pm 6.14$ & $13.26 \pm 1.31$ & $14.77 \pm 0.94$ & $11.90 \pm 1.27$ \\
\hline$\% \mathrm{HCT}$ & $29.00 \pm 12.47$ & $38.40 \pm 5.25$ & $43.77 \pm 2.57$ & $34.31 \pm 4.43$ \\
\hline $\operatorname{MCV}(f \mathrm{f})$ & $44.56 \pm 2.06$ & $47.74 \pm 1.44$ & $47.83 \pm 0.86$ & $46.33 \pm 1.35$ \\
\hline $\mathrm{MCH}(\mathrm{Pg})$ & $12.08 \pm 6.33$ & $16.54 \pm 0.43$ & $16.17 \pm 0.32$ & $16.13 \pm 0.17$ \\
\hline $\mathrm{MCHC}(\mathrm{g} / \mathrm{dL})$ & $27.22 \pm 14.66$ & $34.68 \pm 1.71$ & $33.80 \pm 0.42$ & $34.77 \pm 0.98$ \\
\hline $\operatorname{PLT}\left(10^{9} / \mathrm{L}\right)$ & $545.6 \pm 201.98$ & $819.2 \pm 100.86$ & $878.0 \pm 275.67$ & $626.7 \pm 257.57$ \\
\hline
\end{tabular}

Fig. 2 ATRA delayed TAM-induced cancer initiation in osteosarcoma cells. BALB/c mice were intrafemorally injected with $1 \times 10^{5} \mathrm{~K} 7 \mathrm{M} 2$ WT cells alone or mixed with RAW264.7 cells and sacrificed after 4 weeks to assess tumor formation. a Representative images of osteosarcoma tumor formation. $\mathbf{b}$ The weight of the legs harboring tumors. ${ }^{* *} P<0.001$ (vs. the indicated groups). $\mathbf{c}$ Immunofluorescence analysis of $\mathrm{F} 4 / 80^{+}$ macrophages and CD209 ${ }^{+}$TAMs in the tumors (left). The F4/80 $0^{+}$area (\% of tumor area) and the CD209 ${ }^{+}$area (\% of tumor area) were quantified (right). d Whole blood assay 
a

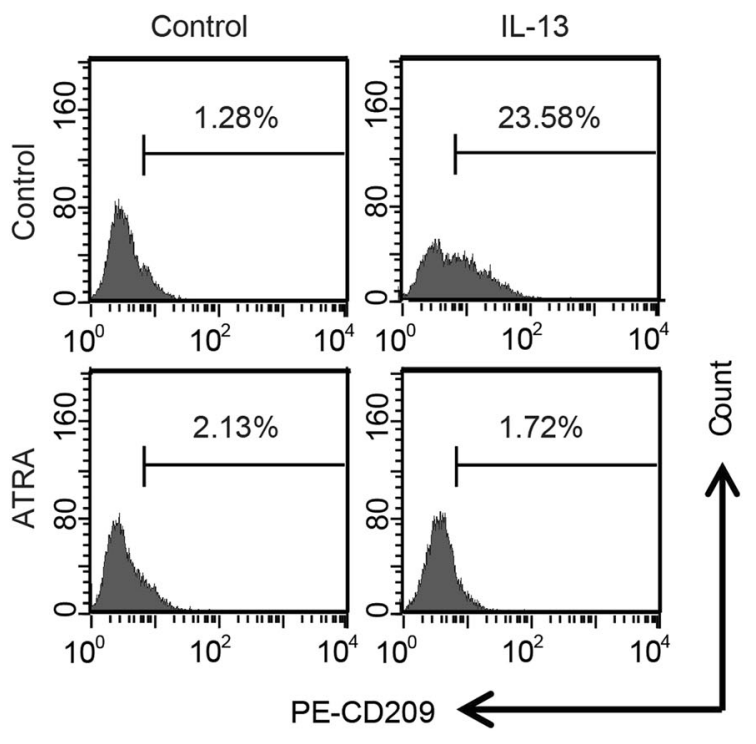

b
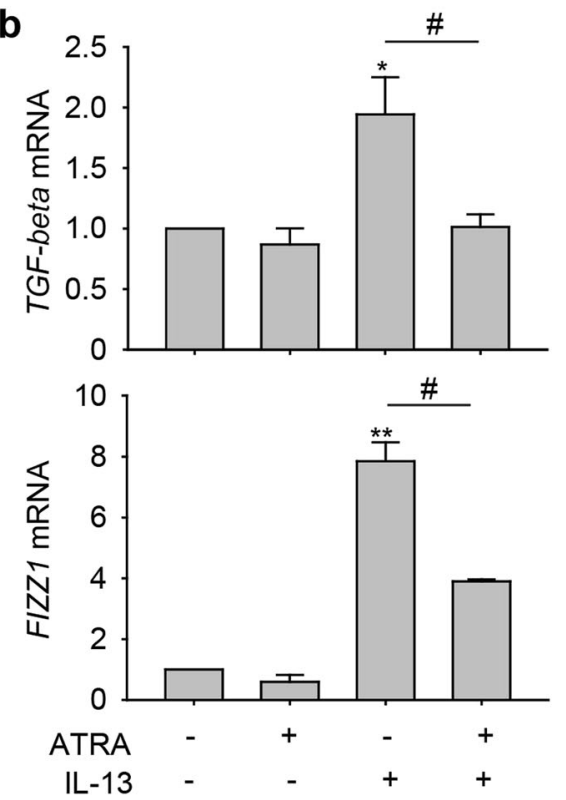

Fig. 3 ATRA inhibited M2 polarization of macrophages. a Flow cytometric analysis of the CD209+ cell population in RAW264.7 cells treated with IL-13 in the absence or presence of $1 \mu \mathrm{M}$ ATRA. $\mathbf{b}$ The mRNA levels of TGF-beta and FIZZ1 in RAW264.7 cells treated with IL-13 alone or in combination with $1 \mu \mathrm{M}$ ATRA. ${ }^{*} P<0.05 ;{ }^{*} P<0.01$ (vs. Control); ${ }^{*} P<0.05$ (vs. Combination)

significantly slower tumor growth promoted by RAW264.7 cells (Fig. 2a, b). ATRA treatment also appreciably reduced the enrichment of F4/80 ${ }^{+} \mathrm{CD} 209^{+}$cells (Fig. 2c). In addition, we also conducted whole blood assays, and the data showed no significant differences among these four groups (Fig. 2d). Thus, we concluded that M2-polarized TAMs could enhance osteosarcoma initiation and that ATRA could delay M2 macrophage-promoted osteosarcoma initiation.

ATRA directly inhibits $M 2$ polarization of macrophages in vitro To confirm that the inhibitory effect of ATRA on M2 macrophagepromoted osteosarcoma initiation was the result of the direct polarization regulation of macrophages, we investigated the influence of ATRA on the polarization of macrophages in vitro. We treated RAW264.7 cells with IL-13 alone to induce M2 polarization of macrophages or in combination with $1 \mu \mathrm{M}$ ATRA and then determined the abundance of $\mathrm{CD}_{209^{+}}$(M2 marker) cells by flow cytometry. As shown in Fig. 3a, the percentage of CD209 ${ }^{+}$ cells increased from $1.28 \%$ of control cells to $23.58 \%$ of IL-13-treated cells, and ATRA markedly decreased the percentage of IL-13-induced CD209+ cells (from $23.58 \%$ in the IL-13 treatment group to $1.72 \%$ in the IL-13 and ATRA combination treatment group). Moreover, we utilized real-time PCR to verify the inhibitory effect of ATRA on M2 polarization of macrophages. The results showed that TGF-beta and FIZZ1 mRNA levels were increased by IL-13 and significantly reduced by ATRA treatment (Fig. 3b). Thus, these data confirmed that ATRA could directly inhibit M2-like polarization of macrophages to regulate osteosarcoma initiation.

ATRA suppresses the colony formation and osteosphere formation abilities of osteosarcoma cells by decreasing M2 polarization Given that M2-polarized TAMs can promote CSC activity to initiate several cancers, we sought to investigate whether ATRA could affect the stemness of osteosarcoma cells by inhibiting M2 polarization. First, we used a colony formation assay to evaluate the proliferation of osteosarcoma cells. The results showed that medium from IL-13- or IL-4-treated macrophages (RM: RAW 264.7 medium; BM: BMDM medium) promoted colony formation by K7M2 WT cells, while RM and BM from cells treated with ATRA in addition to IL-13 or IL-4 significantly inhibited colony formation by K7M2 WT cells (Fig. 4a, b). Then, we analyzed the sphere-forming capability of K7M2 WT cells treated with medium from IL-13- induced M2 macrophages. As shown in Fig. 4c, medium from IL13-induced M2 macrophages effectively enhanced the osteosphere-forming capability, but ATRA markedly reduced this enhancement of osteosphere formation, indicating that ATRA might have the ability to inhibit the CSC properties of osteosarcoma cells promoted by $\mathrm{M} 2$ polarization.

ATRA decreases M2 polarization-induced stemness of osteosarcoma cells

Since CD117 and Stro-1 have been identified as osteosarcoma stem cell markers, we further assessed the expression of CD117/Stro-1 by flow cytometry. We treated K7M2 WT cells with different conditioned media from RAW 264.7 cells (treated with IL-13 alone or in combination with ATRA) and determined the proportion of $\mathrm{CD} 117^{+}$Stro- ${ }^{+}$cells. The results showed that the percentage of CD $117^{+}$Stro- $1^{+}$cells increased from $1.51 \% \pm 0.02 \%$ of control cells to $12.46 \% \pm 3.32 \%$ of $\mathrm{K} 7 \mathrm{M} 2 \mathrm{WT}$ cells when the cells were incubated with IL-13-treated RM (Fig. 5a). Notably, ATRA appreciably decreased the percentage of $\mathrm{CD} 117^{+}$Stro- ${ }^{+}$cells induced by IL-13-treated RM (from $12.46 \% \pm 3.32 \%$ in the IL-13 treatment group to $2.67 \% \pm 1.21 \%$ in the IL-13 and ATRA combination treatment group).

Furthermore, a number of studies have reported that some subpopulations of osteosarcoma cells express prospective CSC markers, including CD133, CXCR4, Nanog, and Oct4 [26-28]. Hence, we next determined the mRNA levels of CD133, CXCR4, Nanog, and Oct4 by real-time PCR. Fig. $5 \mathrm{~b}$ shows that the mRNA expression levels of CD133, CXCR4, Nanog, and Oct4 were higher in both the IL13 and IL-4 treatment groups than in the control group but were reduced by ATRA cotreatment. Taken together, these results further confirmed that ATRA effectively inhibited the stemness of osteosarcoma cells by reducing M2 polarization of TAMs. *

\section{DISCUSSION}

Tumor-associated macrophages directly participate in tumor initiation, progression, and metastasis via numerous mechanisms. In this study, we demonstrated that M2 polarization of TAMs promoted osteosarcoma initiation and stemness, indicating that TAMs might be a promising target for osteosarcoma treatment. Furthermore, our data showed that ATRA inhibited M2 polarization of macrophages and weakened the CSC properties of 
a
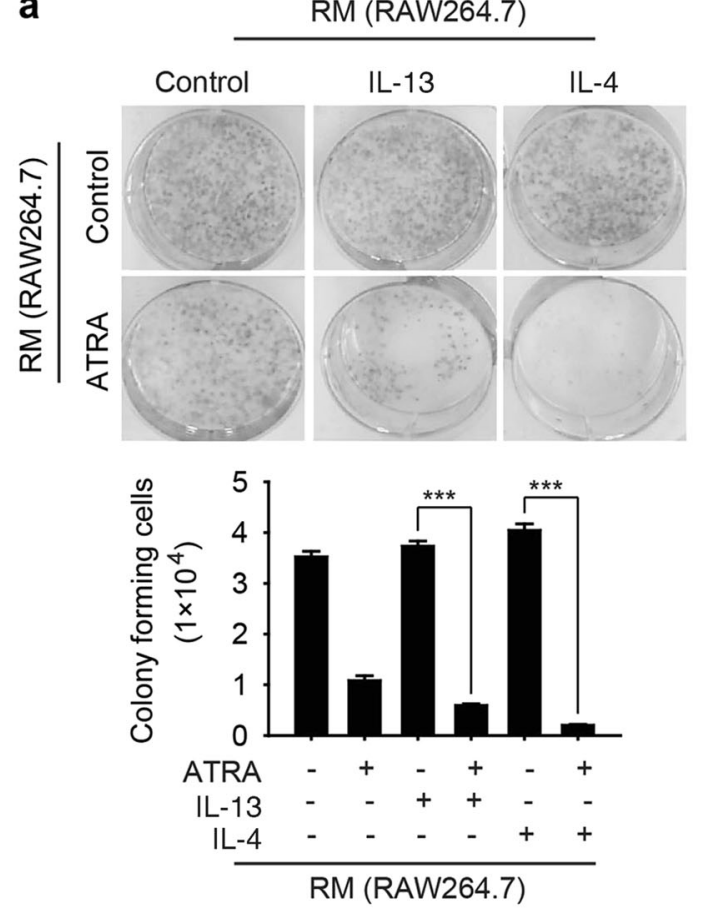

b
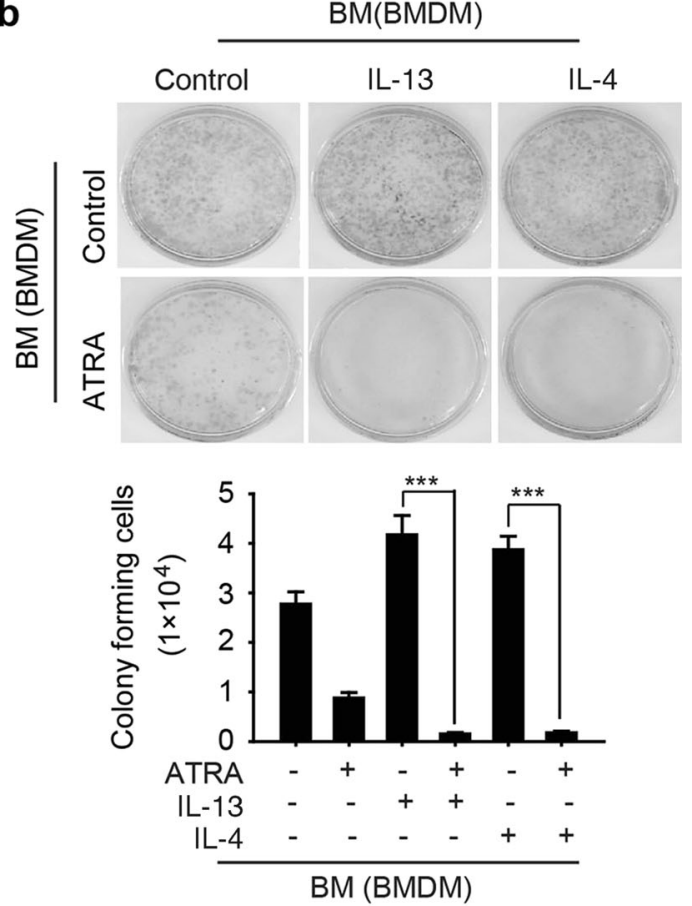

C

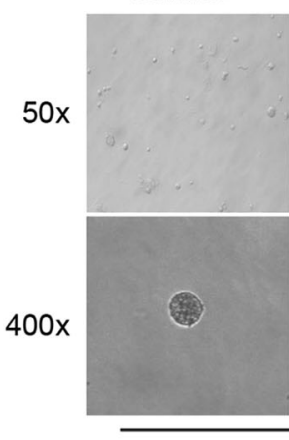

ATRA

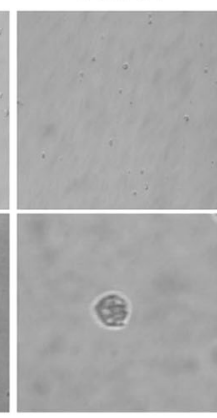

$\mathrm{IL}-13$

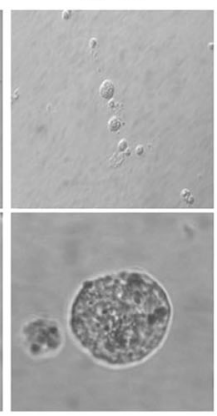

RM (RAW264.7)
IL-13+ATRA
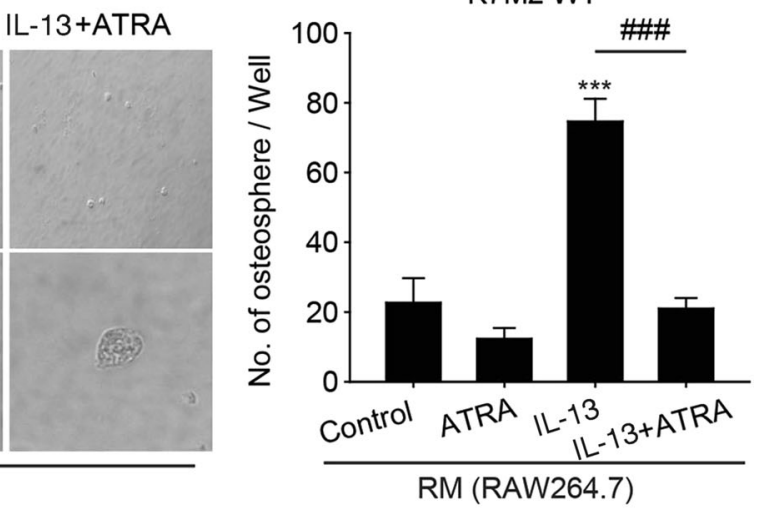

Fig. 4 ATRA suppressed colony formation and osteosphere formation by osteosarcoma cells. a, b Colony formation assay. K7M2 WT cells were treated with RM or BM for 7 days, and the cell colony-forming ability was assessed by a colony formation assay. Above are representative images, and below is the quantitative analysis. ${ }^{* * *} P<0.001$ (vs. the indicated groups). c K7M2 WT cells treated with RM for 14 days were subjected to a tumor sphere-formation assay. Left: representative images of osteospheres. Right: quantification of the assay results. ${ }^{* * *} P<$

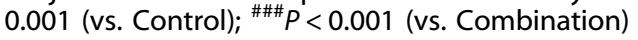

osteosarcoma cells induced by M2-type macrophages, which suggested that ATRA might be a prospective agent for eradicating osteosarcoma via M2 macrophage inhibition.

TAMs serve as a source of key components in inflammatory microenvironments, and increasing evidence indicates that even before cancer begins, chronic inflammation or prolonged inflammatory episodes can set the stage for oncogenesis $[29,30]$. After infiltration into tumors, TAMs contribute to chronic inflammation by secreting inflammatory cytokines, such as IL-1 $\beta$, IL-6, and CXCL8 [31-33]. While TAMs in the preinvasive niche contribute to oncogenic transformation and survival, accumulating evidence suggests that they are also critical for the selfrenewal and maintenance of CSCs in established tumors [15-19]. For example, Mitchem et al. showed that ablation of CCR2 or CSF1R signaling significantly blocked TAM infiltration in pancreatic ductal adenocarcinoma (PDAC), decreased the number of $\mathrm{CD} 44^{+} \mathrm{ALDH} 1^{+} \mathrm{CSCS}$, and improved the response to chemotherapy [18]. In our study, we first demonstrated that M2-type macrophages increase the population of $\mathrm{CD}_{117^{+}}$Stro- $1^{+} \mathrm{CSC}$, accompanied by the increased expression of prospective CSC markers (CD133, CXCR4, Nanog, and Oct4) and promote sphere formation by osteosarcoma cells. Taken together, these results indicate that M2-polarized TAMs significantly accelerate osteosarcoma initiation. Thus, we proposed M2-polarized TAMs as an appropriate therapeutic target for osteosarcoma.

M2 TAMs are an attractive target in biological therapies for osteosarcoma, and preventing cancer initiation and stemness through inhibiting M2-type macrophages might be feasible in osteosarcoma therapy. ATRA was shown to effectively skew macrophages away from M2 polarization in our previous study [20]. In the current study, our data also demonstrated that ATRA attenuated osteosarcoma initiation and stemness via M2 TAM intervention. Researchers have shed new light on the role of ATRA in cancer stem cell inhibition and tumor growth delay. Aldehyde dehydrogenase 1 (ALDH1) is a cancer stem-like cell (CSC) marker in human cancers, and Young et al. reported that ALDH1 activity 


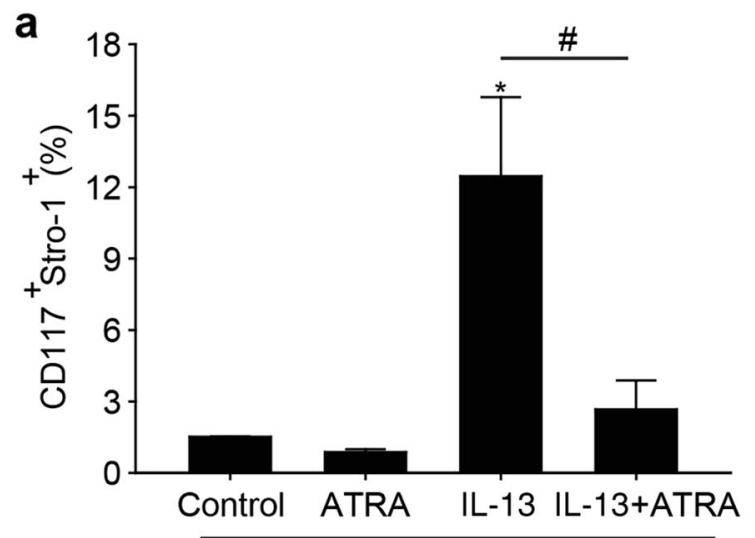

RM (RAW264.7)

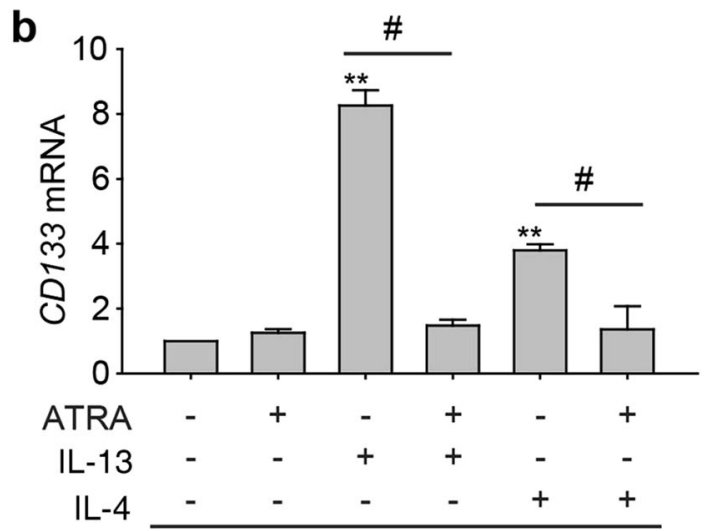

RM (RAW264.7)

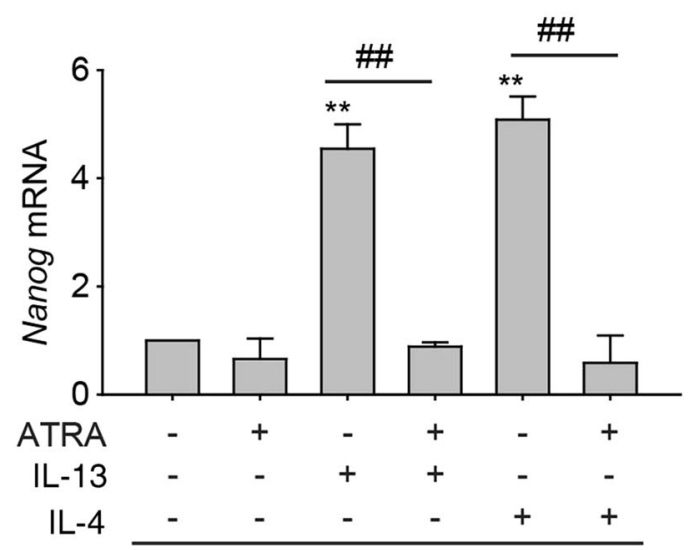

RM (RAW264.7)

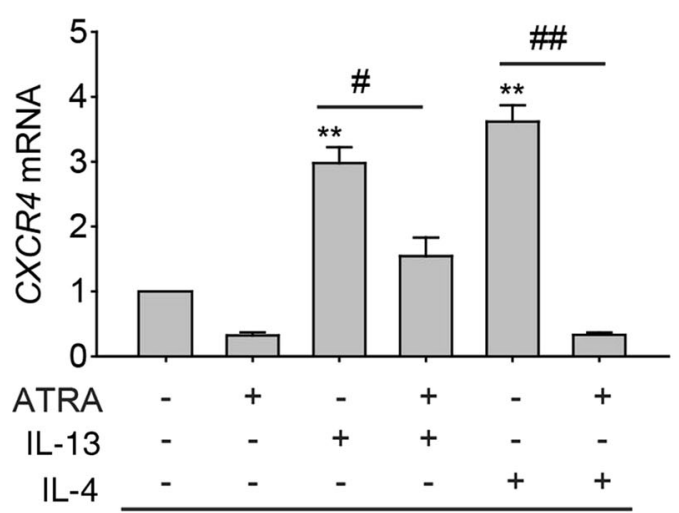

RM (RAW264.7)

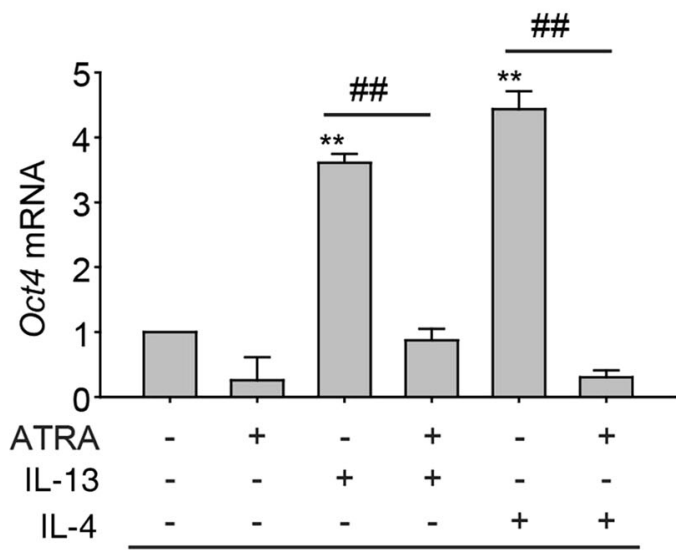

RM (RAW264.7)

Fig. 5 ATRA inhibited the stemness of osteosarcoma cells. a Flow cytometric analysis of the CD117 ${ }^{+}$Stro- $1^{+}$cell population in K7M2 WT cells incubated with RM treated with IL-13 in the absence or presence of $1 \mu \mathrm{M}$ ATRA. ${ }^{*} P<0.05$ (vs. Control); ${ }^{\#} P<0.05$ (vs. Combination). $\mathbf{b}$ The mRNA levels of CD133, CXCR4, Nanog, and Oct4 in K7M2 WT cells incubated with RM treated with IL-13 or IL-4 alone or in combination with $1 \mu$ M

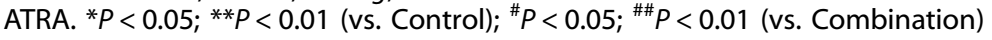

was positively correlated with stemness in ovarian cancer cells as evidenced by properties such as sphere formation and CSC marker expression, as well as by tumorigenesis in a mouse xenograft model, and that ATRA reduced ALDH1 expression, suppressed tumor formation and inhibited sphere formation, cell migration and invasion in ALDH1-abundant ovarian cancer cells [34]. In addition, in patient-derived gastric carcinoma cells with high aldehyde dehydrogenase (ALDH) activity, ATRA inhibited both tumor sphere initiation and growth in vitro, and ATRA treatment was sufficient to inhibit gastric tumor progression in vivo [35]. Hence, CSCs are potential targets for ATRA in cancer therapeutics.

Given that TAMs can enhance tumor progression by directly communicating with CSCs to promote their stemness and/or subsequent oncogenic properties, we proposed the possibility of directly targeting TAMs or disrupting TAM-CSC crosstalk for cancer therapy. In this study, we report that ATRA delays osteosarcoma formation and attributes this effect to the inhibition of M2-type macrophages and decreased CSC formation in osteosarcoma. 
Future research is necessary to explore the underlying mechanism of TAM-CSC coregulation in osteosarcoma progression and uncover mechanisms of ATRA in tumor initiation. In addition, both our study and other previous studies show that ATRA is recognized to have a direct inhibitory effect on osteosarcoma cells by inducing cell differentiation [36, 37]. We previously reported that ATRA prevented osteosarcoma metastasis by inhibiting M2 polarization of TAMs [20]. Taken together, these findings indicate that ATRA has great potential as an anticancer agent in osteosarcoma due to its multiple effects, including inducing cell differentiation, preventing cell metastasis and decreasing CSCs.

In summary, we provide evidence that M2-type macrophages promote osteosarcoma initiation through CSC activation, whereas ATRA prevents tumor formation and stemness by inhibiting M2 macrophage polarization. Our results link TAMs and CSCs to the anticancer effect of ATRA on osteosarcoma. Our findings not only provide a possible mechanism of osteosarcoma initiation but also propose the clinical application of ATRA to target TAM-induced CSCs for osteosarcoma eradication.

\section{ACKNOWLEDGEMENTS}

This work was supported by the State Key Program of the National Natural Science Foundation of China (81830107 to QJH) and by grants from the National Natural Science Foundation of China (81473225 to QJH, 81803552 to XJS, and 81603126 to $\mathrm{NZ}$ ).

\section{AUTHOR CONTRIBUTIONS}

QJH, MDY, QZ, and XJS designed the research project; QZ, XJS, SFX, and YQC performed the experiments; QJH, MDY, QZ, XJS, JC, HZ, and BY analyzed the data; NZ contributed patient samples; and QJH, MDY, QZ, and XJS wrote the paper.

\section{ADDITIONAL INFORMATION}

Competing interests: The authors declare no competing interests.

\section{REFERENCES}

1. Kansara M, Teng MW, Smyth MJ, Thomas DM. Translational biology of osteosarcoma. Nat Rev Cancer. 2014;14:722-35.

2. Gianferante DM, Mirabello L, Savage SA. Germline and somatic genetics of osteosarcoma-connecting aetiology, biology and therapy. Nat Rev Endocrinol. 2017:13:480-91.

3. Pece S, Tosoni D, Confalonieri S, Mazzarol G, Vecchi M, Ronzoni S, et al. Biological and molecular heterogeneity of breast cancers correlates with their cancer stem cell content. Cell. 2010;140:62-73.

4. Todaro M, Francipane MG, Medema JP, Stassi G. Colon cancer stem cells: promise of targeted therapy. Gastroenterology. 2010;138:2151-62.

5. Reya T, Morrison SJ, Clarke MF, Weissman IL. Stem cells, cancer, and cancer stem cells. Nature. 2001;414:105-11.

6. Al-Hajj M, Clarke MF. Self-renewal and solid tumor stem cells. Oncogene. 2004:23:7274-82.

7. Bae JH, Park SH, Yang JH, Yang K, Yi JM. Stem cell-like gene expression signature identified in ionizing radiation-treated cancer cells. Gene. 2015;572:285-91.

8. Korkaya H, Liu S, Wicha MS. Breast cancer stem cells, cytokine networks, and the tumor microenvironment. J Clin Invest. 2011;121:3804-9.

9. Fessler E, Dijkgraaf FE, De Sousa EMF, Medema JP. Cancer stem cell dynamics in tumor progression and metastasis: is the microenvironment to blame? Cancer Lett. 2013;341:97-104.

10. Lonardo E, Frias-Aldeguer J, Hermann PC, Heeschen C. Pancreatic stellate cells form a niche for cancer stem cells and promote their self-renewal and invasiveness. Cell Cycle. 2012;11:1282-90.

11. Lonardo E, Hermann PC, Mueller MT, Huber S, Balic A, Miranda-Lorenzo I, et al. Nodal/Activin signaling drives self-renewal and tumorigenicity of pancreatic cancer stem cells and provides a target for combined drug therapy. Cell Stem Cell. 2011;9:433-46.

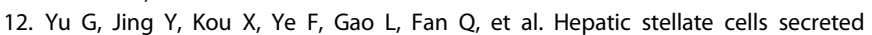
hepatocyte growth factor contributes to the chemoresistance of hepatocellular carcinoma. PLoS ONE. 2013;8:e73312.
13. Noy R, Pollard JW. Tumor-associated macrophages: from mechanisms to therapy. Immunity. 2014:41:49-61.

14. Raggi C, Mousa HS, Correnti M, Sica A, Invernizzi P. Cancer stem cells and tumorassociated macrophages: a roadmap for multitargeting strategies. Oncogene. 2016;35:671-82.

15. Fan QM, Jing YY, Yu GF, Kou XR, Ye F, Gao L, et al. Tumor-associated macrophages promote cancer stem cell-like properties via transforming growth factor-beta1induced epithelial-mesenchymal transition in hepatocellular carcinoma. Cancer Lett. 2014;352:160-8.

16. Yang J, Liao D, Chen C, Liu Y, Chuang TH, Xiang R, et al. Tumor-associated macrophages regulate murine breast cancer stem cells through a novel paracrine EGFR/Stat3/Sox-2 signaling pathway. Stem Cells. 2012;31:248-58.

17. Jinushi M, Chiba S, Yoshiyama H, Masutomi K, Kinoshita I, Dosaka-Akita H, et al. Tumor-associated macrophages regulate tumorigenicity and anticancer drug responses of cancer stem/initiating cells. Proc Natl Acad Sci USA. 2011;108:12425-30.

18. Mitchem JB, Brennan DJ, Knolhoff BL, Belt BA, Zhu Y, Sanford DE, et al. Targeting tumor-infiltrating macrophages decreases tumor-initiating cells, relieves immunosuppression, and improves chemotherapeutic responses. Cancer Res. 2013;73:1128-41.

19. Nusblat LM, Carroll MJ, Roth CM. Crosstalk between M2 macrophages and glioma stem cells. Cell Oncol (Dordr). 2017;40:471-82.

20. Zhou Q, Xian M, Xiang S, Xiang D, Shao X, Wang J, et al. All-trans retinoic acid prevents osteosarcoma metastasis by inhibiting M2 polarization of tumorassociated macrophages. Cancer Immunol Res. 2017;5:547-59.

21. Fan TM. Animal models of osteosarcoma. Expert Rev Anticancer Ther. 2010;10:1327-38.

22. Yao Z, Zhang J, Zhang B, Liang G, Chen X, Yao F, et al. Imatinib prevents lung cancer metastasis by inhibiting M2-like polarization of macrophages. Pharm Res. 2018;133:121-31.

23. Tariq M, Zhang JQ, Liang GK, He QJ, Ding L, Yang B. Gefitinib inhibits M2-like polarization of tumor-associated macrophages in Lewis lung cancer by targeting the STAT6 signaling pathway. Acta Pharmacol Sin. 2017;38:1501-11.

24. Xu T, Huang C, Qi XT, Yang XC, Zhang N, Cao J, et al. 2-Bromopalmitate sensitizes osteosarcoma cells to adriamycin-induced apoptosis via the modulation of CHOP. Eur J Pharmacol. 2018;844:204-15.

25. Qi XT, Li YL, Zhang YQ, Xu T, Lu B, Fang L, et al. KLF4 functions as an oncogene in promoting cancer stem cell-like characteristics in osteosarcoma cells. Acta Pharmacol Sin. 2019;40:546-55.

26. Gatti M, Solari A, Pattarozzi A, Campanella C, Thellung S, Maniscalco L, et al. In vitro and in vivo characterization of stem-like cells from canine osteosarcoma and assessment of drug sensitivity. Exp Cell Res. 2018;363:48-64

27. Huynh DL, Kwon T, Zhang JJ, Sharma N, Gera M, Ghosh M, et al. Wogonin suppresses stem cell-like traits of CD133 positive osteosarcoma cell via inhibiting matrix metallopeptidase-9 expression. BMC Complement Alter Med. 2017;17:304.

28. Paiva-Oliveira DI, Martins-Neves SR, Abrunhosa AJ, Fontes-Ribeiro C, Gomes CMF. Therapeutic potential of the metabolic modulator metformin on osteosarcoma cancer stem-like cells. Cancer Chemother Pharmacol. 2018;81:49-63.

29. Moran EM. Epidemiological and clinical aspects of nonsteroidal anti-inflammatory drugs and cancer risks. J Environ Pathol Toxicol Oncol. 2002;21:193-201.

30. Kortylewski M, Xin H, Kujawski M, Lee H, Liu Y, Harris T, et al. Regulation of the IL23 and IL-12 balance by Stat 3 signaling in the tumor microenvironment. Cancer Cell. 2009;15:114-23.

31. Balkwill F, Mantovani A. Inflammation and cancer: back to Virchow? Lancet. 2001;357:539-45.

32. Waugh DJ, Wilson C. The interleukin-8 pathway in cancer. Clin Cancer Res. 2008;14:6735-41.

33. Fang W, Ye L, Shen L, Cai J, Huang F, Wei Q, et al. Tumor-associated macrophages promote the metastatic potential of thyroid papillary cancer by releasing CXCL8. Carcinogenesis. 2014;35:1780-7.

34. Young MJ, Wu YH, Chiu WT, Weng TY, Huang YF, Chou CY. All-trans retinoic acid downregulates ALDH1-mediated stemness and inhibits tumour formation in ovarian cancer cells. Carcinogenesis. 2015;36:498-507.

35. Nguyen PH, Giraud J, Staedel C, Chambonnier L, Dubus P, Chevret E, et al. Alltrans retinoic acid targets gastric cancer stem cells and inhibits patient-derived gastric carcinoma tumor growth. Oncogene. 2016;35:5619-28.

36. Ying $M$, Zhang L, Zhou Q, Shao X, Cao J, Zhang N, et al. The E3 ubiquitin protein ligase MDM2 dictates all-trans retinoic acid-induced osteoblastic differentiation of osteosarcoma cells by modulating the degradation of RARalpha. Oncogene. 2016;35:4358-67.

37. Yang QJ, Zhou LY, Mu YQ, Zhou QX, Luo JY, Cheng L, et al. All-trans retinoic acid inhibits tumor growth of human osteosarcoma by activating Smad signalinginduced osteogenic differentiation. Int J Oncol. 2012;41:153-60. 